\title{
ON THE OPTIMALITY SYSTEM FOR A 1-D EULER FLOW PROBLEM
}

\author{
Eugene M. Cliff* Matthias Heinkenschloss ${ }^{\dagger}$ \\ Ajit R. Shenoy $\ddagger$ \\ Interdisciplinary Center for Applied Mathematics \\ Virginia Tech \\ Blacksburg, Virginia 24061
}

\begin{abstract}
$\underline{\text { Abstract }}$
In this paper, a problem of shape design for a duct with the flow governed by the one-dimensional Euler equations is analyzed. The flow is assumed to be transonic, in the sense that we have a shock embedded in the flow. The presence of the shock introduces analytical and numerical difficulties that are overcome by introducing the shock location as an explicit state variable. We justify the use of adjoint variables by establishing conditions to ensure that the linearized constraint is surjective. These adjoint variables are used to state necessary optimality conditions and to compute gradients. In addition, our characterization of the adjoint variables is of interest for comparison to adjoints associated with shock-capturing formulations.
\end{abstract}

\section{$\underline{\text { 1. Introduction }}$}

In this paper we apply the shock-fitting framework developed in Cliff et $a l^{3}$ to an optimal duct design problem governed by the one-dimensional Euler equations. The objective of this research is the rigorous mathematical justification of differentiability of the problem, surjectivity of the linearized constraints, and the accurate formulation of the adjoint equations for optimality. The purposes of this analysis are two-fold: first, as the foundation for the efficient numerical solution of the problem by an all-at-once method, and; second, for comparison to the behavior of adjoints arising from more formal treatments, including those arising from shock-capturing procedures.

\footnotetext{
*Reynolds Metal Professor, Aerospace Engineering Department, Associate Fellow AIAA

$\dagger$ Assistant Professor, Mathematics Department

$\ddagger$ Graduate Student, Aerospace Engineering Department, Student Member AIAA
}

The following notation will be used:

$$
\begin{aligned}
\rho & =\text { density } \\
u & =\text { velocity, } \\
e & =\text { specific total energy, } \\
h & =\text { height of the channel, } \\
q & =h_{x} / h \text { logarithmic derivative of channel height, } \\
\gamma & =\text { specific heat ratio, } \\
p & =(\gamma-1) \rho\left(e-\frac{u^{2}}{2}\right) \quad \text { pressure. }
\end{aligned}
$$

A quasi-one-dimensional inviscid steady state flow can be described by the steady state Euler equations in conservation form

$$
[\mathbf{F}(\mathbf{U}(x))]_{x}+q(x) \mathbf{Q}(\mathbf{U}(x))=\mathbf{0}, \quad 0 \leq x \leq 1
$$

where

$$
\begin{gathered}
\mathbf{U}=\left(\begin{array}{c}
\mathbf{U}_{1} \\
\mathbf{U}_{2} \\
\mathbf{U}_{3}
\end{array}\right)=\left(\begin{array}{c}
\rho \\
\rho u \\
\rho e
\end{array}\right), \\
\mathbf{F}(\mathbf{U})=\left(\begin{array}{c}
\rho u \\
p+\rho u^{2} \\
(\rho e+p) u
\end{array}\right), \quad \mathbf{Q}(\mathbf{U})=\left(\begin{array}{c}
\rho u \\
\rho u^{2} \\
(\rho e+p) u
\end{array}\right) .
\end{gathered}
$$

See e.g. Anderson. ${ }^{1}$ It should be noted that, that $\mathbf{F}$ and $\mathbf{Q}$ are homogeneous of degree one, i.e. $\mathbf{F}(t \mathbf{U})=t \mathbf{F}(\mathbf{U})$ and $\mathbf{Q}(t \mathbf{U})=t \mathbf{Q}(\mathbf{U})$ for all $t \in \mathbb{R}$. One can easily deduce that

$$
\mathbf{F}(\mathbf{U})=\mathbf{A}(\mathbf{U}) \mathbf{U} \quad \text { and } \quad \mathbf{Q}(\mathbf{U})=\mathbf{S}(\mathbf{U}) \mathbf{U}
$$


where

$$
\begin{gathered}
\mathbf{A}(\mathbf{U})=\frac{d \mathbf{F}(\mathbf{U})}{d \mathbf{U}} \\
=\left(\begin{array}{ccc}
0 & 1 & 0 \\
\frac{\gamma-3}{2} u^{2} & (3-\gamma) u & \gamma-1 \\
-\gamma u e+(\gamma-1) u^{3} & \gamma e-3 \frac{\gamma-1}{2} u^{2} & \gamma u
\end{array}\right)
\end{gathered}
$$

and

$$
\begin{gathered}
\mathbf{S}(\mathbf{U})=\frac{d \mathbf{Q}(\mathbf{U})}{d \mathbf{U}} \\
=\left(\begin{array}{ccc}
0 & 1 & 0 \\
-u^{2} & 2 u & 0 \\
-\gamma u e+(\gamma-1) u^{3} & \gamma e-3 \frac{\gamma-1}{2} u^{2} & \gamma u
\end{array}\right) .
\end{gathered}
$$

It can be shown that $\mathbf{A}(\mathbf{U})$ has eigenvalues $u-a, u$, $u+a$, where $a$ is the speed of sound. Boundary conditions for the system will be specified below. Subsequently we will often omit the argument $x$.

The problem we are interested in is the design of an area function $h$ generating a flow that best approximates a desired pressure distribution $p^{d}$ in the least squares sense. We assume that the area function $h$ is fixed at some point, say $x=0$. This allows us to use the logarithmic derivative $q$ of $h$ as the design parameter. For given $q(x)=h^{\prime}(x) / h(x)$ the area function can be computed from

$$
h(x)=\exp \left(\ln (h(0))+\int_{0}^{x} q(t) d t\right) .
$$

The mathematical formulation of our design problem is as follows:

$$
\operatorname{Minimize} J(\mathbf{U}, q)=\frac{1}{2} \int_{0}^{1}\left(p(x)-p^{d}(x)\right)^{2} d x
$$

subject to the steady state Euler equations

$$
[\mathbf{F}(\mathbf{U})]_{x}+q \mathbf{Q}(\mathbf{U})=\mathbf{0}, \quad 0 \leq x \leq 1,
$$

The flow is assumed to be transonic, with the shock implicitly defined by the Rankine Hugoniot condition,

$$
\mathbf{F}\left(\mathbf{U}\left(x_{s}-\right)\right)=\mathbf{F}\left(\mathbf{U}\left(x_{s}+\right)\right) \text {. }
$$

We have boundary conditions at the inlet

$$
\mathbf{B}^{i}(\mathbf{U}(0))=\left(\begin{array}{c}
\rho \\
\rho u \\
\rho e
\end{array}\right)-\left(\begin{array}{c}
\rho_{\text {in }} \\
(\rho u)_{\text {in }} \\
(\rho e)_{\text {in }}
\end{array}\right)=\mathbf{0},
$$

and boundary conditions at the outlet

$$
\begin{aligned}
B^{o}(\mathbf{U}) & =p-p_{\text {out }}=0 \\
& =(\gamma-1)\left[\mathbf{U}_{3}-\frac{\mathbf{U}_{2}^{2}}{2 \mathbf{U}_{1}}\right]-p_{\text {out }}
\end{aligned}
$$

In addition, we impose bounds on the logarithmic derivative of the area function

$$
0 \leq q_{\text {low }} \leq q(x) \leq q_{\text {upp }}, \quad x \in[0,1] .
$$

Iollo et $a l^{6}$ have studied this problem using an adjoint method to formulate the optimality conditions. The adjoint system derived by them differs from that in the current paper. The boundary conditions imposed on the adjoint variables at the shock by Iollo et $a l^{6}$ seem to be incorrect, except in a special case. This will be discussed in greater detail in Section 4. An extension of their results to the two-dimensional case is considered in Iollo and Salas. ${ }^{7}$ Jameson $^{8}$ has also used an adjoint method to formulate a similar class of optimal design problems.

A similar problem has been studied by Frank and Shubin. ${ }^{5}$ They point out that solutions of this simple model exhibit phenomena quite similar to those in two dimensional inviscid flow over an airfoil. They have shown that under given assumptions, the continuity and energy equations can be integrated out reducing the Euler system to a single ODE. In their version of the problem, a desired velocity profile is set as a target instead of a pressure distribution. They discuss numerical solutions for this problem, employing standard discretization schemes, and using both a black-box method and a Sequential Quadratic Programming (SQP) method. They have reported that the SQP scheme is not very robust, as compared to the black-box scheme, though when it does converge, it proves to be the superior method. Similar results, using the SQP method, have been reported in Sadchikova et al. ${ }^{11}$ The failure of the SQP method stems from the existence of the shock in the flow. In particular, when Godunov-type schemes with low continuity properties are used to capture the shock, we find that the SQP method, which is designed for smooth problems, performs poorly.

In previous research ${ }^{3}$ we discussed an approach to overcome this problem by treating the shock location as an explicit state variable and enforcing the shock condition as an additional state constraint. This was done in the context of the single ODE model described by Frank and Shubin. ${ }^{5}$ We found that this approach yields better smoothness properties for the constraints. We provided a rigorous mathematical analysis of the existence and 
nature of optimal solutions for the infinite-dimensional problem, and studied the relation between the infinitedimensional and the discrete problem, which revealed valuable insight into improving the performance of the optimization algorithm.

Other aspects of this design problem and other methods for its solution have been studied by Borggaard, ${ }^{2}$ by Shenoy and Cliff ${ }^{12}$ and by Narducci, Grossman, and Haftka. ${ }^{10}$ In Borggaard ${ }^{2}$ the flow variables are viewed as functions of the design variables and a sensitivity equation approach is used to compute the gradient. An optimal control approach is used by Shenoy and Cliff. ${ }^{12}$ They assumed that the shock location is known. The area and the flow velocity are the state variables. The design problem is then formulated as an optimal control problem governed by a system of ODEs and solved using a multiple shooting method for the two-point boundary value problem which is obtained from the necessary optimality conditions. In Narducci et $a l^{10}$ sensitivities for various (semi-) discretizations are studied. Although the shock location is treated implicitly, it enters the discretization scheme for the objective function. The design problem is solved numerically using the black-boxapproach with a steepest descent method.

\section{The Design Problem in the Transformed Domain}

For a rigorous mathematical treatment of the optimal control problem we transform the design problem presented in the introduction. In this transformation, the shock location is introduced as an explicit variable and the regions left and right of the shock are transformed onto the fixed interval $[0,1]$ :

$$
\begin{aligned}
{\left[0, x_{s}\right] } & \longrightarrow[0,1] \\
\mathbf{U}(x) & \longrightarrow \mathbf{U}\left(x_{s} \xi\right)
\end{aligned}
$$

and

$$
\begin{aligned}
{\left[1, x_{s}\right] } & \longrightarrow[0,1] \\
\mathbf{U}(x) & \longrightarrow \mathbf{U}\left(1-\left(1-x_{s}\right) \xi\right)
\end{aligned}
$$

With we find that the Euler equations (7), the Rankine Hugoniot shock conditions (8) and the boundary conditions (9), (10) are transformed into the following system of equations for $\mathbf{U}_{l}$ and $\mathbf{U}_{r}$ :

$$
\begin{aligned}
{\left[\mathbf{F}\left(\mathbf{U}_{l}\right)\right]_{\xi}+x_{s} q_{l} \mathbf{Q}\left(\mathbf{U}_{l}\right) } & =\mathbf{0}, \quad \xi \in[0,1], \\
{\left[\mathbf{F}\left(\mathbf{U}_{r}\right)\right]_{\xi}+\left(x_{s}-1\right) q_{r} \mathbf{Q}\left(\mathbf{U}_{r}\right) } & =\mathbf{0}, \quad \xi \in[0,1],
\end{aligned}
$$

with Rankine Hugoniot shock condition

$$
\mathbf{F}\left(\mathbf{U}_{l}(1)\right)=\mathbf{F}\left(\mathbf{U}_{r}(1)\right)
$$

and boundary conditions

$$
\mathbf{B}^{i}\left(\mathbf{U}_{l}(0)\right)=\mathbf{0}
$$

at the inlet and with boundary conditions

$$
B^{o}\left(\mathbf{U}_{r}(0)\right)=0
$$

at the outlet. For convenience, we summarize these conditions in a composite constraint function

$$
\begin{aligned}
& \mathbf{C}\left(\mathbf{U}_{l}, \mathbf{U}_{r}, x_{s}, q_{l}, q_{r}\right)= \\
& \left(\begin{array}{c}
{\left[\mathbf{F}\left(\mathbf{U}_{l}\right)\right]_{\xi}+x_{s} q_{l} \mathbf{Q}\left(\mathbf{U}_{l}\right)} \\
{\left[\mathbf{F}\left(\mathbf{U}_{r}\right)\right]_{\xi}+\left(x_{s}-1\right) q_{r} \mathbf{Q}\left(\mathbf{U}_{r}\right)} \\
\mathbf{F}\left(\mathbf{U}_{l}(1)\right)-\mathbf{F}\left(\mathbf{U}_{r}(1)\right) \\
\mathbf{B}^{i}\left(\mathbf{U}_{l}(0)\right) \\
B^{o}\left(\mathbf{U}_{r}(0)\right)
\end{array}\right)
\end{aligned}
$$

With this formulation the system (13-17) can be written as:

$$
\mathbf{C}\left(\mathbf{U}_{l}, \mathbf{U}_{r}, x_{s}, q_{l}, q_{r}\right)=\mathbf{0}
$$

The systems (7-10) and (13-17) are equivalent. If $\mathbf{U}$ is a solution of the original system (7-10) with shock location $x_{s}$, then the solution of the transformed system (13-17) can be computed from (12). On the other hand, if $\left(\mathbf{U}_{l}, \mathbf{U}_{r}, x_{s}\right)$ is a solution to the transformed system (13-17), then the solution $\mathbf{U}$ to the original system can be computed from

$$
\mathbf{U}(x)= \begin{cases}\mathbf{U}_{l}\left(\frac{x}{x_{s}}\right), & x \in\left[0, x_{s}\right), \\ \mathbf{U}_{r}\left(\frac{1-x}{1-x_{s}}\right), & x \in\left[x_{s}, 1\right] .\end{cases}
$$

To formulate the control problem for the transformed problem, we define the desired pressures

$$
\begin{aligned}
& p_{l}^{d}\left(\xi ; x_{s}\right)=p^{d}\left(x_{s} \xi\right) \\
& p_{r}^{d}\left(\xi ; x_{s}\right)=p^{d}\left(1-\left(1-x_{s}\right) \xi\right) .
\end{aligned}
$$


With the transformations (12), (20) the objective function (6) is given by

$$
\begin{array}{r}
\int_{0}^{1}\left(p(x)-p^{d}(x)\right)^{2} d x=\int_{0}^{x_{s}}\left(p(x)-p^{d}(x)\right)^{2} d x \\
+\int_{x_{s}}^{1}\left(p(x)-p^{d}(x)\right)^{2} d x \\
=x_{s} \int_{0}^{1}\left(p_{l}(\xi)-p_{l}^{d}\left(\xi ; x_{s}\right)\right)^{2} d \xi \\
+\left(1-x_{s}\right) \int_{0}^{1}\left(p_{r}(\xi)-p_{r}^{d}\left(\xi ; x_{s}\right)\right)^{2} d \xi
\end{array}
$$

The control problem we have to solve is given as follows:

$$
\begin{aligned}
& \text { Minimize } J\left(\mathbf{U}_{l}, \mathbf{U}_{r}, x_{s}, q_{l}, q_{r}\right) \\
& \quad=\frac{x_{s}}{2} \int_{0}^{1}\left(p_{l}(\xi)-p_{l}^{d}\left(\xi ; x_{s}\right)\right)^{2} d \xi \\
& +\frac{1-x_{s}}{2} \int_{0}^{1}\left(p_{r}(\xi)-p_{r}^{d}\left(\xi ; x_{s}\right)\right)^{2} d \xi
\end{aligned}
$$

subject to the equality constraints

$$
\mathbf{C}\left(\mathbf{U}_{l}, \mathbf{U}_{r}, x_{s}, q_{l}, q_{r}\right)=\mathbf{0}
$$

and to the inequality constraints

$$
\begin{gathered}
0 \leq x_{s} \leq 1, \\
0 \leq q_{\text {low }} \leq q_{l}(\xi), q_{r}(\xi) \leq q_{\text {upp }}, \quad \xi \in[0,1] .
\end{gathered}
$$

The states are given by the triple $\left(\mathbf{U}_{l}, \mathbf{U}_{r}, x_{s}\right)$ and the controls are $\left(q_{l}, q_{r}\right)$. The equations (13), (14), (15), (16), (17) are the state equations. The flow solutions $\left(\mathbf{U}_{l}, \mathbf{U}_{r}\right)$ and the design functions $\left(q_{l}, q_{r}\right)$ do not depend on the shock location explicitly; they are coupled to shock location $x_{s}$ only through the state equations (13), (14), (15).

For a complete description of the control problem, we have to specify the control space and the state space. It will be seen that an appropriate choice for the control space is

$$
\mathcal{Q}=L^{\infty}(0,1) \times L^{\infty}(0,1) .
$$

The set of admissible parameters is defined by

$$
\mathcal{Q}_{a d}=\left\{\left(q_{l}, q_{r}\right) \in \mathcal{Q} \mid q_{\mathrm{low}} \leq q_{l}(\xi), q_{r}(\xi) \leq q_{\mathrm{upp}}\right\}
$$

for $\xi \in[0,1]$. Recall that $q_{\text {low }} \geq 0$. A typical control vector is denoted by

$$
\mathbf{q} \equiv\left(q_{l}, q_{r}\right) .
$$

The state space is chosen to be

$$
\mathcal{V}=\mathbf{W}^{1, \infty}(0,1) \times \mathbf{W}^{1, \infty}(0,1) \times \mathbb{R},
$$

where $\mathbf{W}^{1, \infty}(0,1)=\left(W^{1, \infty}(0,1)\right)^{3}$ and a typical vector is denoted as

$$
\mathbf{v} \equiv\left(\mathbf{U}_{l}, \mathbf{U}_{r}, x_{s}\right) .
$$

Defining the space

$$
\mathcal{C}=\mathbf{L}^{\infty}(0,1) \times \mathbf{L}^{\infty}(0,1) \times \mathbb{R}^{7},
$$

with $\mathbf{L}^{\infty}(0,1)=\left(L^{\infty}(0,1)\right)^{3}$, the constraint, (18), is viewed as a map from $\mathcal{V} \times \mathcal{Q}$ into $\mathcal{C}$ :

$$
\mathbf{C}: \mathcal{V} \times \mathcal{Q} \rightarrow \mathcal{C}
$$

For later purposes we also define the adjoint space

$$
\boldsymbol{\Lambda}=\mathbf{W}^{1, \infty}(0,1) \times \mathbf{W}^{1, \infty}(0,1) \times \mathbb{R}^{7} .
$$

As usual, for a function $f:[0,1] \rightarrow \mathbb{R}$ we set

$$
\begin{aligned}
\|f\|_{\infty} & =\operatorname{ess}_{\sup _{[0,1]}|f(\xi)|,} \\
\|f\|_{1, \infty} & =\|f\|_{\infty}+\left\|f_{\xi}\right\|_{\infty} .
\end{aligned}
$$

For a vector valued function $\mathbf{F}$ we define $\|\mathbf{F}\|_{\infty},\|\mathbf{F}\|_{1, \infty}$ analogously.

\section{Differentiability and Surjectivity}

The transformation of the design problem performed in Section 2 allows us to establish the Fréchet differentiability of the constraint function $\mathbf{C}$ and of the objective function $J$. The proofs for Fréchet differentiability are very similar to those presented in Cliff $e t \mathrm{al}^{3}$ for the Frank and Shubin model problem. In the following we make use of the notation (3), (4) and (29).

Theorem 3.1 The nonlinear operator $\mathbf{C}: \mathcal{V} \times \mathcal{Q} \rightarrow \mathcal{C}$ is Fréchet differentiable at all $(\mathbf{v}, \mathbf{q}) \in \mathcal{V} \times \mathcal{Q}_{\text {ad }}$ with $\rho_{l}, \rho_{r}>0$. The partial Fréchet derivatives are given by

$$
\begin{aligned}
& \mathbf{C}_{\mathbf{v}}(\mathbf{v}, \mathbf{q})(\widetilde{\mathbf{v}}) \\
& =\left(\begin{array}{c}
{\left[\mathbf{A}\left(\mathbf{U}_{l}\right) \widetilde{\mathbf{U}}_{l}\right]_{\xi}+x_{s} q_{l} \mathbf{S}\left(\mathbf{U}_{l}\right) \widetilde{\mathbf{U}}_{l}+\widetilde{x}_{s} q_{l} \mathbf{Q}\left(\mathbf{U}_{l}\right)} \\
{\left[\mathbf{A}\left(\mathbf{U}_{r}\right) \widetilde{\mathbf{U}}_{r}\right]_{\xi}+\left(x_{s}-1\right) q_{r} \mathbf{S}\left(\mathbf{U}_{r}\right) \widetilde{\mathbf{U}}_{r}+\widetilde{x}_{s} q_{r} \mathbf{Q}\left(\mathbf{U}_{r}\right)} \\
\mathbf{A}\left(\mathbf{U}_{l}(1)\right) \widetilde{\mathbf{U}}_{l}(1)-\mathbf{A}\left(\mathbf{U}_{r}(1)\right) \widetilde{\mathbf{U}}_{r}(1) \\
\frac{d}{d U} \mathbf{B}^{i}\left(\mathbf{U}_{l}(0)\right) \widetilde{\mathbf{U}}_{l}(0) \\
\frac{d}{d U} B^{o}\left(\mathbf{U}_{r}(0)\right) \widetilde{\mathbf{U}}_{r}(0)
\end{array}\right),
\end{aligned}
$$


where $\frac{d}{d \mathbf{U}} \mathbf{B}^{i}\left(\mathbf{U}_{l}(0)\right)=\mathbf{I}_{3 \times 3}$ is equal to the identity, $\widetilde{\mathbf{v}}=$ $\left(\widetilde{\mathbf{U}}_{l}, \widetilde{\mathbf{U}}_{r}, \widetilde{x}_{s}\right)$ and $^{1}$

$$
\begin{aligned}
& \frac{d}{d \mathbf{U}} B^{o}\left(\mathbf{U}_{r}(0)\right)=\frac{d p_{r}(0)}{d \mathbf{U}} \\
& \quad=\left(\frac{\gamma-1}{2} u_{r}^{2}(0),-(\gamma-1) u_{r}(0), \gamma-1\right),
\end{aligned}
$$

and

$$
\mathbf{C}_{\mathbf{q}}(\mathbf{v}, \mathbf{q})(\widetilde{\mathbf{q}})=\left(\begin{array}{c}
x_{s} \mathbf{Q}\left(\mathbf{U}_{l}\right) \widetilde{q}_{l} \\
\left(x_{s}-1\right) \mathbf{Q}\left(\mathbf{U}_{r}\right) \widetilde{q}_{r} \\
\mathbf{0} \\
\mathbf{0} \\
0
\end{array}\right)
$$

where $\widetilde{\mathbf{q}}=\left(\widetilde{q}_{l}, \widetilde{q}_{r}\right)$.

Proof: The proof of this result is similar to the corresponding one in Cliff $e t a l^{3}$ and is therefore omitted.

Theorem 3.2 If the desired pressure $p^{d}$ is differentiable with absolutely continuous derivative, then the objective function $J$ is Fréchet differentiable. The partial Fréchet -derivatives are given by

$$
\begin{gathered}
J_{\mathbf{U}_{l}}(\mathbf{v}, \mathbf{q}) \widetilde{\mathbf{U}}_{l}=x_{s} \int_{0}^{1}\left(p_{l}-p_{l}^{d}\right) \frac{d p_{l}}{d \mathbf{U}_{l}} \widetilde{\mathbf{U}}_{l} d \xi \\
J_{\mathbf{U}_{r}}(\mathbf{v}, \mathbf{q}) \widetilde{\mathbf{U}}_{r}= \\
\left(1-x_{s}\right) \int_{0}^{1}\left(p_{r}-p_{r}^{d}\right) \frac{d p_{r}}{d \mathbf{U}_{r}} \widetilde{\mathbf{U}}_{r} d \xi
\end{gathered}
$$

where $\frac{d p_{i}}{d \mathbf{U}_{i}}=\left(\frac{\gamma-1}{2} u_{i}^{2},-(\gamma-1) u_{i}, \gamma-1\right), i=l, r, c f$. (33), and

$$
\begin{aligned}
J_{x_{s}}(\mathbf{v}, \mathbf{q}) & \\
& =\int_{0}^{1} \frac{1}{2}\left(p_{l}(\xi)-p_{l}^{d}\left(\xi ; x_{s}\right)\right)^{2} d \xi \\
& -\int_{0}^{1} x_{s}\left(p_{l}(\xi)-p_{l}^{d}\left(\xi ; x_{s}\right)\right)\left(p_{l}^{d}\right)_{x}(\xi) \xi d \xi \\
& -\int_{0}^{1} \frac{1}{2}\left(p_{r}(\xi)-p_{r}^{d}\left(\xi ; x_{s}\right)\right)^{2} d \xi \\
& +\int_{0}^{1}\left(1-x_{s}\right)\left(p_{r}(\xi)-p_{r}^{d}\left(\xi ; x_{s}\right)\right)\left(p_{r}^{d}\right)_{x}(\xi) \xi d \xi .
\end{aligned}
$$

\footnotetext{
${ }^{1}$ In the following, we interpret $\frac{d p_{r}(0)}{d \mathrm{U}}$ as a row or a column, as
} appropriate. The form should be apparent from the context.
Proof: The assertion follows from the definition of $J$ using standard estimates. The proof is therefore omitted.

The final result of this section concerns the surjectivity of the linearized constraints. This property is important for the formulation of optimality conditions, see e.g. Maurer and Zowe. ${ }^{9}$ Due to the partitioning of variables into states and controls, the surjectivity of the linearization of $\mathbf{C}$ is guaranteed if $\mathbf{C}_{\mathbf{v}}(\mathbf{v}, \mathbf{q})$ is surjective. Recall that $a_{l}$ and $a_{r}$ denote the speed of sound in the regions left and right of the shock, respectively.

Theorem 3.3 Let $\left(\mathbf{U}_{l}, \mathbf{U}_{r}, x_{s}\right)$ be given with $u_{l}(\xi)>0$, $u_{l}(\xi) \neq a_{l}(\xi), u_{r}(\xi)>0, u_{r}(\xi) \neq a_{r}(\xi)$ for all $\xi \in$ $[0,1]$ and $x_{s} \in[0,1]$. If

$$
\begin{gathered}
\left(\mathbf{A}\left(\mathbf{U}_{r}(0)\right)^{-T} \frac{d p_{r}(0)}{d \mathbf{U}}\right)^{T} \int_{0}^{1}\left[\mathbf{T}_{r} q_{r} \mathbf{Q}\left(\mathbf{U}_{r}\right)\right. \\
\left.-\mathbf{T}_{r}(1) \mathbf{T}_{l}(1)^{-1} \mathbf{T}_{l} q_{l} \mathbf{Q}\left(\mathbf{U}_{l}\right)\right] d \zeta \neq 0
\end{gathered}
$$

where

$$
\begin{aligned}
\mathbf{T}_{l}(\xi) & =\exp \left(\int_{0}^{\xi} x_{s} q_{l}(t) \mathbf{S}\left(\mathbf{U}_{l}(t)\right) \mathbf{A}\left(\mathbf{U}_{l}(t)\right)^{-1} d t\right), \\
\mathbf{T}_{r}(\xi) & =\exp \left(\int_{0}^{\xi}\left(x_{s}-1\right) q_{r}(t) \mathbf{S}\left(\mathbf{U}_{r}(t)\right) \mathbf{A}\left(\mathbf{U}_{r}(t)\right)^{-1} d t\right),
\end{aligned}
$$

then for all $\mathbf{r}=\left(\mathbf{r}_{l}, \mathbf{r}_{r}, \mathbf{r}_{s}, \mathbf{r}_{\mathrm{in}}, r_{\text {out }}\right) \in \mathcal{C}$ the linear system

$$
\mathbf{C}_{\mathbf{v}}(\mathbf{v}, \mathbf{q})(\widetilde{\mathbf{v}})=\mathbf{r}
$$

admits a unique solution $\widetilde{\mathbf{v}}$.

Proof: Under the assumptions of the theorem, the matrices $\mathbf{A}\left(\mathbf{U}_{l}\right)$ and $\mathbf{A}\left(\mathbf{U}_{r}\right)$ are invertible for all $\xi \in[0,1]$. Therefore, the first equation in (39) is equivalent to

$$
\begin{gathered}
{\left[\mathbf{A}\left(\mathbf{U}_{l}\right) \widetilde{\mathbf{U}}_{l}\right]_{\xi}+x_{s} q_{l} \mathbf{S}\left(\mathbf{U}_{l}\right) \mathbf{A}\left(\mathbf{U}_{l}\right)^{-1} \mathbf{A}\left(\mathbf{U}_{l}\right) \widetilde{\mathbf{U}}_{l}} \\
+\widetilde{x}_{s} q_{l} \mathbf{Q}\left(\mathbf{U}_{l}\right)=\mathbf{r}_{l} .
\end{gathered}
$$

Using the integrating factor $\mathbf{T}_{l}$ the solution can shown to be

$$
\begin{gathered}
\widetilde{\mathbf{U}}_{l}(\xi)=\mathbf{A}\left(\mathbf{U}_{l}(\xi)\right)^{-1} \mathbf{T}_{l}(\xi)^{-1}\left[\mathbf{A}\left(\mathbf{U}_{l}(0)\right) \widetilde{\mathbf{U}}_{l}(0)\right. \\
\left.+\int_{0}^{\xi} \mathbf{T}_{l}(\zeta)\left(\mathbf{r}_{l}(\zeta)-\widetilde{x}_{s} q_{l}(\zeta) \mathbf{Q}\left(\mathbf{U}_{l}(\zeta)\right)\right) d \zeta\right]
\end{gathered}
$$

Similarly, the solution to the second equation

$$
\begin{gathered}
{\left[\mathbf{A}\left(\mathbf{U}_{r}\right) \widetilde{\mathbf{U}}_{r}\right]_{\xi}+\left(x_{s}-1\right) q_{r} \mathbf{S}\left(\mathbf{U}_{r}\right) \widetilde{\mathbf{U}}_{r}} \\
+\widetilde{x}_{s} q_{r} \mathbf{Q}\left(\mathbf{U}_{r}\right)=\mathbf{r}_{r}
\end{gathered}
$$


can be computed using the integrating factor $\mathbf{T}_{r}$ and is given by

$$
\begin{gathered}
\widetilde{\mathbf{U}}_{r}(\xi)=\mathbf{A}\left(\mathbf{U}_{r}(\xi)\right)^{-1} \mathbf{T}_{r}(\xi)^{-1}\left[\mathbf{A}\left(\mathbf{U}_{r}(0)\right) \widetilde{\mathbf{U}}_{r}(0)\right. \\
\left.+\int_{0}^{\xi} \mathbf{T}_{r}(\zeta)\left(\mathbf{r}_{r}(\zeta)-\widetilde{x}_{s} q_{r}(\zeta) \mathbf{Q}\left(\mathbf{U}_{r}(\zeta)\right)\right) d \zeta\right]
\end{gathered}
$$

Inserting (40) and (41) into the third equation $\mathbf{A}\left(\mathbf{U}_{l}(1)\right) \widetilde{\mathbf{U}}_{l}(1)-\mathbf{A}\left(\mathbf{U}_{r}(1)\right) \widetilde{\mathbf{U}}_{r}(1)=\mathbf{r}_{s}$ yields

$$
\begin{aligned}
\widetilde{x}_{s} \int_{0}^{1}\left[\mathbf{T}_{r}(1)^{-1} \mathbf{T}_{r}(\zeta) q_{r}(\zeta) \mathbf{Q}\left(\mathbf{U}_{r}(\zeta)\right)\right. \\
\left.-\mathbf{T}_{l}(1)^{-1} \mathbf{T}_{l}(\zeta) q_{l}(\zeta) \mathbf{Q}\left(\mathbf{U}_{l}(\zeta)\right)\right] d \zeta \\
=\mathbf{r}_{s}-\mathbf{T}_{l}(1)^{-1}\left[\mathbf{A}\left(\mathbf{U}_{l}(0)\right) \widetilde{\mathbf{U}}_{l}(0)\right. \\
\left.+\int_{0}^{1} \mathbf{T}_{l}(\zeta) \mathbf{r}_{l}(\zeta) d \zeta\right] \\
+\mathbf{T}_{r}(1)^{-1}\left[\mathbf{A}\left(\mathbf{U}_{r}(0)\right) \widetilde{\mathbf{U}}_{r}(0)\right. \\
\left.+\int_{0}^{1} \mathbf{T}_{r}(\zeta) \mathbf{r}_{r}(\zeta) d \zeta\right]
\end{aligned}
$$

Next, we have to incorporate the equations arising from the boundary conditions. The equation $\frac{d}{d \mathbf{U}} \mathbf{B}^{i}\left(\mathbf{U}_{l}(0)\right) \widetilde{\mathbf{U}}_{l}(0)=\mathbf{r}_{\text {in }}$ implies $\widetilde{\mathbf{U}}_{l}(0)=\mathbf{r}_{\text {in }}$. If $\mathbf{w}_{\mathbf{1}}, \mathbf{w}_{\mathbf{2}}$ are linearly independent vectors orthogonal to $\frac{d p_{r}(0)}{d \mathbf{U}}$, then $\frac{d}{d \mathbf{U}} B^{o}\left(\mathbf{U}_{l}(0)\right) \widetilde{\mathbf{U}}_{r}(0)=r_{\text {out }}$ implies that

$$
\widetilde{\mathbf{U}}_{r}(0)=\frac{r_{\text {out }}}{\left\|\frac{d p_{r}(0)}{d \mathbf{U}}\right\|^{2}} \frac{d p_{r}(0)}{d \mathbf{U}}+\omega_{1} \mathbf{w}_{\mathbf{1}}+\omega_{2} \mathbf{w}_{\mathbf{2}}
$$

Notice that, since $\gamma \neq 1$, the inequality $\left\|\frac{d p_{r}(0)}{d \underline{\widetilde{U}}}\right\|^{2}>0$ holds true. Inserting these expressions for $\widetilde{\mathbf{U}}_{l}(0)$ and $\widetilde{\mathbf{U}}_{r}(0)$ into (42) shows that the existence of a unique solution of (39), i.e. the existence of a unique solution $\left(\widetilde{x}_{s}, \omega_{1}, \omega_{2}\right)$, can be guaranteed if the $3 \times 3$ matrix

$$
\begin{gathered}
\left(\int _ { 0 } ^ { 1 } \left[\mathbf{T}_{r}(1)^{-1} \mathbf{T}_{r} q_{r} \mathbf{Q}\left(\mathbf{U}_{r}\right)\right.\right. \\
\left.\quad-\mathbf{T}_{l}(1)^{-1} \mathbf{T}_{l} q_{l} \mathbf{Q}\left(\mathbf{U}_{l}\right)\right] d \zeta \\
-\mathbf{T}_{r}(1)^{-1} \mathbf{A}\left(\mathbf{U}_{r}(0)\right) \mathbf{w}_{\mathbf{1}} \\
\left.-\mathbf{T}_{r}(1)^{-1} \mathbf{A}\left(\mathbf{U}_{r}(0)\right) \mathbf{w}_{\mathbf{2}}\right)
\end{gathered}
$$

is nonsingular. This is the case if and only if

$$
\left(\mathbf { A } ( \mathbf { U } _ { r } ( 0 ) ) ^ { - 1 } \int _ { 0 } ^ { 1 } \left[\mathbf{T}_{r} q_{r} \mathbf{Q}\left(\mathbf{U}_{r}\right)\right.\right.
$$

$\left.-\mathbf{T}_{r}(1) \mathbf{T}_{l}(1)^{-1} \mathbf{T}_{l} q_{l} \mathbf{Q}\left(\mathbf{U}_{l}\right)\right] d \zeta$

$$
\left.-\mathbf{w}_{1},-\mathbf{w}_{2}\right)
$$

is nonsingular. Since $\mathbf{w}_{\mathbf{1}}, \mathbf{w}_{\mathbf{2}}$ are linearly independent vectors orthogonal to $\frac{d p_{r}(0)}{d \mathbf{U}}$, the latter condition is equivalent to (38).

\section{Optimality Conditions}

We define the vector

$$
\eta \equiv\left(\boldsymbol{\lambda}_{l}, \boldsymbol{\lambda}_{r}, \boldsymbol{\lambda}_{s}, \boldsymbol{\lambda}_{i}, \lambda_{o}\right) \in \boldsymbol{\Lambda}
$$

and the Lagrange function

$$
\begin{aligned}
\mathcal{L}(\mathbf{v}, \eta) & \\
= & \frac{x_{s}}{2} \int_{0}^{1}\left(p_{l}-p_{l}^{d}\right)^{2} d \xi \\
& +\frac{1-x_{s}}{2} \int_{0}^{1}\left(p_{r}-p_{r}^{d}\right)^{2} d \xi \\
& +\int_{0}^{1} \boldsymbol{\lambda}_{l}^{T}\left[\left(\mathbf{F}\left(\mathbf{U}_{l}\right)\right)_{\xi}+x_{s} q_{l} \mathbf{Q}\left(\mathbf{U}_{l}\right)\right] d \xi \\
& +\int_{0}^{1} \boldsymbol{\lambda}_{r}^{T}\left[\left(\mathbf{F}\left(\mathbf{U}_{r}\right)\right)_{\xi}+\left(x_{s}-1\right) q_{r} \mathbf{Q}\left(\mathbf{U}_{r}\right)\right] d \xi \\
& +\boldsymbol{\lambda}_{s}^{T}\left[\mathbf{F}\left(\mathbf{U}_{l}(1)\right)-\mathbf{F}\left(\mathbf{U}_{r}(1)\right)\right] \\
& +\boldsymbol{\lambda}_{i}^{T} \mathbf{B}^{i}\left(\mathbf{U}_{l}(0)\right)+\lambda_{o} B^{o}\left(\mathbf{U}_{r}(0)\right) .
\end{aligned}
$$

If the shock location at the optimum obeys $x_{s} \in(0,1)$, then the first order necessary optimality conditions are

$$
\begin{aligned}
& \mathbf{0}=\mathcal{L}_{\mathbf{v}}(\mathbf{v}, \mathbf{q}, \eta)(\widetilde{\mathbf{v}}) \\
& \mathbf{0}=\mathcal{L}_{\eta}(\mathbf{v}, \mathbf{q}, \eta)(\widetilde{\eta}) \\
& 0 \leq \mathcal{L}_{\mathbf{q}}(\mathbf{v}, \mathbf{q}, \eta)(\widetilde{\mathbf{q}})
\end{aligned}
$$

for all $\widetilde{\mathbf{v}} \in \mathcal{C}$, for all $\widetilde{\eta} \in \boldsymbol{\Lambda}$, and for all $\widetilde{\mathbf{q}}$ with $(\mathbf{q}+\widetilde{\mathbf{q}}) \in$ $\mathcal{Q}_{a d}$.

The second equation (45) in the optimality system yields the state system (13-17). Using integration by parts and appropriate test functions $\widetilde{\mathbf{U}}_{l}, \widetilde{\mathbf{U}}_{r}, \widetilde{x}_{s}$ we find that the first equation (44) in the optimality system yields the adjoint equations

$$
\left[\mathbf{A}\left(\mathbf{U}_{l}\right)\right]^{T}\left(\boldsymbol{\lambda}_{l}\right)_{\xi}
$$




$$
\begin{aligned}
& =x_{s} q_{l}\left[\mathbf{S}\left(\mathbf{U}_{l}\right)\right]^{T} \boldsymbol{\lambda}_{l}+x_{s}\left(p_{l}-p_{l}^{d}\right)\left[\frac{d p_{l}}{d \mathbf{U}_{l}}\right] \\
& {\left[\mathbf{A}\left(\mathbf{U}_{r}\right)\right]^{T}\left(\boldsymbol{\lambda}_{r}\right)_{\xi}} \\
& =\left(x_{s}-1\right) q_{r}\left[\mathbf{S}\left(\mathbf{U}_{r}\right)\right]^{T} \boldsymbol{\lambda}_{r} \\
& \quad+\left(1-x_{s}\right)\left(p_{r}-p_{r}^{d}\right)\left[\frac{d p_{r}}{d \mathbf{U}_{r}}\right]
\end{aligned}
$$

with conditions

$$
\lambda_{l}(1)=-\lambda_{s}, \quad \lambda_{r}(1)=\lambda_{s}
$$

at the shock, the boundary conditions

$$
\begin{aligned}
& \lambda_{i}^{T} \frac{d}{d \mathbf{U}} \mathbf{B}^{i}\left(\mathbf{U}_{l}(0)\right)=\lambda_{l}(0)^{T} \mathbf{A}\left(\mathbf{U}_{l}(0)\right) \\
& \lambda_{o} \frac{d}{d \mathbf{U}} B^{o}\left(\mathbf{U}_{r}(0)\right)=\lambda_{r}(0)^{T} \mathbf{A}\left(\mathbf{U}_{r}(0)\right)
\end{aligned}
$$

and the condition

$$
\int_{0}^{1} q_{l} \boldsymbol{\lambda}_{l}^{T} \mathbf{Q}\left(\mathbf{U}_{l}\right)+q_{r} \boldsymbol{\lambda}_{r}^{T} \mathbf{Q}\left(\mathbf{U}_{r}\right) d \xi+J_{x_{s}}(\mathbf{v}, \mathbf{q})=0 .
$$

Using the definitions (9) and (10) one can see that the first equation in (50) is equivalent to

$$
\left[\mathbf{A}\left(\mathbf{U}_{l}(0)\right)\right]^{T} \boldsymbol{\lambda}_{l}(0)=\boldsymbol{\lambda}_{i}
$$

which implies that the Lagrange multipliers $\lambda_{l}$ are free at the left end point $\xi=0$. If we denote the components of $\boldsymbol{\lambda}_{r}$ by $\boldsymbol{\lambda}_{r}^{(1)}, \boldsymbol{\lambda}_{r}^{(2)}, \boldsymbol{\lambda}_{r}^{(3)}$, then the second equation in (50) is equivalent to

$$
\begin{aligned}
& \frac{\gamma-3}{2} u_{r}^{2}(0) \boldsymbol{\lambda}_{r}^{(2)}(0)+\left[-\gamma u_{r}(0) e_{r}(0)\right. \\
& \left.\quad+(\gamma-1) u_{r}^{3}(0)\right] \boldsymbol{\lambda}_{r}^{(3)}(0)=\lambda_{o} \frac{\gamma-1}{2} u_{r}^{2}(0), \\
& \boldsymbol{\lambda}_{r}^{(1)}(0)+(3-\gamma) u_{r}(0) \boldsymbol{\lambda}_{r}^{(2)}(0)+\left[\gamma e_{r}(0)\right. \\
& \left.\quad-3 \frac{\gamma-1}{2} u_{r}^{2}(0)\right] \boldsymbol{\lambda}_{r}^{(3)}(0)=-\lambda_{o}(\gamma-1) u_{r}(0), \\
& (\gamma-1) \boldsymbol{\lambda}_{r}^{(2)}(0)+\gamma u_{r}(0) \boldsymbol{\lambda}_{r}^{(3)}(0) \\
& \quad=\lambda_{o}(\gamma-1)
\end{aligned}
$$

The third equation (46) in the optimality system is equivalent to

$$
x_{s} \boldsymbol{\lambda}_{l}(\xi)^{T} \mathbf{Q}\left(\mathbf{U}_{l}\right) \begin{cases}\geq 0 & \text { if } q_{l}(\xi)=q_{\mathrm{low}}, \\ =0 & \text { if } q_{l}(\xi) \in\left(q_{\mathrm{low}}, q_{\mathrm{upp}}\right), \\ \leq 0 & \text { if } q_{l}(\xi)=q_{\mathrm{upp}}\end{cases}
$$

and

$\left(x_{s}-1\right) \boldsymbol{\lambda}_{r}(\xi)^{T} \mathbf{Q}\left(\mathbf{U}_{r}\right) \begin{cases}\geq 0 & \text { if } q_{r}(\xi)=q_{\mathrm{low}} \\ =0 & \text { if } q_{r}(\xi) \in\left(q_{\text {low }}, q_{\mathrm{upp}}\right) . \\ \leq 0 & \text { if } q_{r}(\xi)=q_{\mathrm{upp}}\end{cases}$

Note the shock condition (49) for the co-state. From the examination of the other equations it can be seen that $\lambda_{l}(1)=-\lambda_{r}(1)=\mathbf{0}$ can be guaranteed only if the desired pressures can be matched exactly, i.e. the source terms in (47), (48), and the term $\mathcal{J}_{x_{s}}\left(\mathbf{U}_{l}, \mathbf{U}_{r}, x_{s}, q_{l}, q_{r}\right)$ in (51) vanish. This homogeneity of the co-states at the shock was assumed generally in Iollo et al. ${ }^{6}$ However, the rigorous presentation shows that the adjoints $\left(\boldsymbol{\lambda}_{l}, \boldsymbol{\lambda}_{r}\right)$ will in general be nonzero at the shock with opposite signs left and right of the shock. The fact that the costates are nonzero at the shock if the optimal value of the objective function is nonzero can also be observed in the numerical experiments for the Frank and Shubin problem reported in Cliff $e t a l .^{3}$

\section{$\underline{\text { 5. Conclusions and Outlook }}$}

We have provided a careful derivation of an optimality system for a shape-design problem based on 1-D Euler flows. The analysis shows that the adjoints are smooth (indeed in $\mathbf{W}^{1, \infty}$ ) in regions where the flow is smooth, but just as with the flow variables, there is a shock in the adjoint variables.

The conditions derived in Section 4 can be used for numerical implementation in several ways. The statemodel (13-17) defines a boundary-value problem to be solved for the flow variables and the shock-location given the area-distribution. With the state/control in-hand, the adjoint system $(47-49,51,53)$ defines the adjoint variables $\left(\boldsymbol{\lambda}_{l}, \boldsymbol{\lambda}_{r}\right)$. Finally, the optimality conditions are given as $(54,55)$. A simple gradient method would proceed by solving the first of these for the state, the second for the adjoint and then using an increment in the control as derived from the optimality conditions. As a globalization strategy, one might use the control increment as a search direction in the control space $\mathcal{Q}$ and perform a line-search for improvement of the cost function. This would require solving the system (22) for the variables $\left(\mathbf{U}_{l}, \mathbf{U}_{r}, x_{s}\right)$ for each trial control increment.

As an alternative approach one might use an SQP framework, wherein the states and controls are treated as independent variables and the state model (22) is treated as an explicit constraint. In this all-at-once framework iterates proceed toward optimality and feasibility at the same time. The rigorous analysis of differentiability and 
existence of Lagrange multipliers is expected to be important here, as it was in Cliff et al. ${ }^{3}$ The study in Frank and Shubin ${ }^{5}$ showed that formal development of an SQP algorithm did not lead to a satisfactory computational procedure. This is because the shock-capturing flow-model used does not possess the necessary smoothness properties to justify Lagrangian formulation. In Cliff $e^{2} \mathrm{al}^{3}$ we utilized the same shock-fitting formulation employed here, but for the single ODE model of the flow. ${ }^{5}$ In the present paper we have applied this approach to the full 1-D Euler system. Additionally, it is worth noting that care is needed in the formulation of the finite-dimensional approximation to preserve consistency with the continous problem. In Cliff et $a l^{3}$ we observed that a modification of the finite-dimensional state/adjoint inner-product had a substantial impact on numerical efficiency of the overall procedure.

The analysis leads to a characterization of optimal shapes and can be numerically implemented in several ways. The flow formulation can be interpreted as a shock-fitting scheme and so is of questionable practical utiltity as a computational procedure for 3-dimensional flows. On the other hand, the analysis clearly reveals the nature of a shock in the adjoint system and this is of value for comparison to shock-capturing methods. There is no reason to believe that a discretization that leads to accurate shock modeling for the flow system is necessarily appropriate for modeling the shock in the adjoint system. The results in this paper can be used to construct test examples since we have a characterization of the adjoint system without numerical artifacts, such as artificial viscosity.

Acknowledgement

This research was supported by the Air Force Office of Scientific Research under grant F49620-93-1-0280 and by the NSF under Grant DMS-9403699.

\section{$\underline{\text { References }}$}

[1] J. D. Anderson. Modern Compressible Flow with Historical Prespective. McGraw-Hill Series in Aeronautical and Aerospace Engineering. McGraw-Hill, New York, 1990.

[2] Borggaard, J. T., The Sensitivity Equation Method for Optimal Design, $\mathrm{PhD}$ thesis, Virginia Polytechnic Institute and State University, Department of Mathematics, Blacksburg, VA 24061-0123, USA, 1994.
[3] Cliff, E.M., Heinkenschloss, M., and Shenoy, A., "Optimal Control for Flows with Discontinuities," Technical Report 95-09-02, Interdisciplinary Center for Applied Mathematics, Virginia Polytechnic Institute and State University, Blacksburg, VA 24061, 1995, also Journal of Optimization, Theory and Application, to appear.

[4] Dennis, J. E., Heinkenschloss, M., and Vicente, L. N., "Trust-region interior-point algorithms for a class of nonlinear programming problems," Technical Report 94-12-01, Interdisciplinary Center for Applied Mathematics, Virginia Polytechnic Institute and State University, Blacksburg, VA 24061, 1994.

[5] Frank, P. D. and Shubin, G. Y., "A comparison of optimization-based approaches for a model computational aerodynamics design problem," J. Comput. Physics, vol. 98, 1992, pp 74-89.

[6] Iollo, A., Salas, M. D. and Ta'asan, S., "Shape Optimization governed by the Euler Equations using an Adjoint Method,' ICASE Report No. 93-78, also NASA CR 191555, November 1993.

[7] Iollo, A. and Salas, M. D., "Contribution to the Optimal Shape Design of Two-Dimensional Internal Flows with Embedded Shocks," ICASE Report No. 95-20, also NASA CR 195062, March 1995.

[8] Jameson, A., "Optimum Aerodynamic Design Using CFD and Control Theory," In AIAA Paper 95 1729, presented at the 12th Computational Fluid Dynamics Conference, San Diego, California, June 1995.

[9] Maurer, H. and Zowe, J. "First and Secondorder necessary and sufficient optimality conditions for infinite-dimensional programming problems," Mathematical Programming, vol. 16, 1979, pp 98110.

[10] Narducci, R., Grossman, B., and Haftka, R. T., "Sensitivity algorithms for an inverse design problem involving a shock wave," In AIAA Paper 940096, presented at the 32nd Aerospace Sciences Meeting \& Exhibit, Reno, Nevada, January 10-13, 1994.

[11] Sadchikova, E., Shenoy, A. and Cliff, E.M., "Computational Issues in Optimization-Based Design," In Proceedings from the 34th IEEE Conference on Decision and Control, New Orleans, December 1995, pp 445-450. 
[12] Shenoy, A. and Cliff, E.M., "An optimal control formulation for a flow matching problem," In Proceedings from the 5th AIAA/USAF/NASA/ISSMO Symposium On Multidisciplinray Analysis And Optimization, Panama City Beach, September 7-9, 1994, pp 520-528. 